\title{
AVALIAÇÃO DO ESTILO, PERSONALIDADE E FOCO NA ATUAÇÃO DO SUPERVISOR DE ESTÁGIOS CLÍNICOS
}

\author{
Luiz Fernando de Lara CAMPOS
}

\begin{abstract}
RESUMO
A avaliação do supervisor de estágios em Psicologia Clínica foi a meta deste estudo. Para elaboração do perfil de atuação do supervisor, foram avaliados o tipo de personalidade, estilo e foco de cada supervisor. Foram utilizados como instrumentos o Inventário de Estilos de Supervisão e o Questionário sobre Foco e Estilo de Supervisão. Os sujeitos foram divididos em dois grupos em razão do enfoque teórico: Cognitivo-Comportamental $(\mathrm{N}=6)$ e Psicodinâmico $(N=12)$. No geral, os resultados demonstraram semelhança quanto ao estilo, foco e personalidade na supervisão.

Palavras-chave: Supervisão Clínica; Estilo de Supervisão; Foco de Supervisão
\end{abstract}

\begin{abstract}
The evaluation of supervisors working on Clinical Psychology was the aim of this study. In order to elaborate the action profile of supervisors' models the type of personality, style and focus was studied. The instruments used were the Supervisor Style Inventory and Supervisory Focus and Style Questionnaire. Subjects were divided in two groups according to their theorical approach used: Cognitive-Behavioral $(N=6)$ and Psychodynamic $(N=12)$. The results showed similarity in relation to style, focus, and personality.
\end{abstract}

Key words: Clinical Supervision; Supervision Style; Supervision Focus

A formação do psicólogo é complexa, pois envolve aspectos não apenas da condição intelectual do estudante, mas também de um sistema de treinamento de uma habilidade específica: a capacidade terapêutica. Neste sentido, o estágio supervisionado é o principal elemento central no treinamento do futuro psicólogo, uma vez que é neste momento que, via de regra, se tem a oportunidade de colocar em prática a maioria dos constructos teóricos estudados nos anos de formação precedente (Campos, 1989; 1994).

A literatura específica neste campo é extremamente rica em opções teóricas e práti- 
cas para todos os supervisores e pesquisadores interessados na temática, mas ainda demonstra falhas quanto a sua ordem e continuidade, mesmo se considerado o acréscimo de produção e interesse nos últimos anos (Campos, 1994).

A supervisão é considerada essencial para a aprendizagem da psicoterapia (Kauderer e Herron, 1990). Talvez esta grande valência da supervisão ocorra em função de que um dos maiores desafios para supervisor e supervisionado é a aprendizagem e o ensino de Psicologia aplicada e, em especial, a prática psicoterápica (Dodds, 1986). Já Robiner e Schofield (1990) afirmam que a supervisão é a parte constante na formação do psicólogo clínico, sendo utilizada igualmente em outras áreas, como na área industrial, escolar, organizacional, entre outras. A posição sobre a supervisão tem se tornado cada vez mais crítica nos últimos anos, levando a um aumento de interesse sobre o tema (Efstation, Patton e Kardash, 1990), com uma especial preocupação com os mecanismos deste processo. Entretanto, Berg e Stone (1980) relatam que dentro da literatura, a situação sobre a validade da supervisão é ambígua, uma vez que existem relatos de trabalhos que indicam esta estratégia como elemento fundamental para a aquisição de habilidades clínicas e outros que a indicam como algo desnecessário ao processo de aprendizagem da prática clínica.

As limitações e dificuldades existentes nos estudos sobre a supervisão, tem, segundo Worthington (1984), suas raízes em três aspectos: (a) falta de uma teoria geral, abrangente sobre o processo de supervisão, (b) aplicação e generalização demasiada dos princípios da aprendizagem social (Bandura,1977) à supervisão e (c) limitações metodológicas em razão de amostras restritas de sujeitos nos estudos realizados. A autora escreve que a supervisão é percebida como efetiva à medida que o supervisor demonstra aceitação e suporte ao supervisionado, ensinando a conceituar, intervir e outras habilidades de modo preciso. $\mathrm{O}$ supervisor deve assumir diversos papéis no processo de supervisão, alternando-os diante da necessidade do supervisionado. O quadro encontrado sugere a mudança de comportamento dos supervisores para que estes se sintonizem com as necessidades de seus supervisionados.

A carência de estudos na área, a dificuldade de se modificar as práticas existentes e a dificuldade de assumir novos papéis, são aspectos que limitam a atuação do supervisor. Vale ressaltar que outra limitação emergente nesta questão é a origem do modelo de supervisão utilizado pela maioria dos supervisores, que parece estar diretamente relacionado ao modelo teórico inerente à sua prática (Bernard e Goodyear, 1992).

Campos (1989) escreve que os supervisores no Brasil ainda trabalham nos moldes do início do século, desconhecendo os avanços desta matéria, e não possuindo nenhuma formação específica e/ou complementar para o exercício da função, Deste modo, a qualidade do ensino e da supervisão fica limitada a conceitos e atitudes pessoais ao invés de um corpo coeso de princípios e objetivos, fato este que pode limitar sensivelmente a aprendizagem, a formação de atitudes e a assimilação de comportamentos éticos.

O ponto vital para esta questão parece ser o modelo de supervisão adotado pelo supervisor (Campos, 1994), uma vez que estes modelos são, em geral, baseados nas próprias teorias psicoterápicas, o que acaba por limitar seu campo de aplicação e validade.

Os modelos tradicionais ou convencionais de supervisão partem da premissa que as instruções diretas fornecidas pelo supervisor ao supervisionado são possíveis de serem executadas durante o atendimento (Feixas, 1992), fato que nem sempre é verdadeiro. Estes modelos não respeitam o supervisionado enquanto ser autônomo e auto-organizado, que está baseado em conceitos que podem permitir, ou não, compreender as próprias instruções. En- 
tretanto, pouco se conhece sobre as variáveis que permeiam este processo, como fica claro nos trabalhos de Stoltemberge Delworth (1988) e Holloway (1988), os quais afirmam que a literatura está repleta de modelos de supervisão gerados pelo senso comum e pela intuição, o que, inicialmente, foi um bom começo, mas claramente já não é suficiente face ao já conhecido. Holloway (1988) afirmou que se deve estabelecer diferenças claras entre os modelos de desenvolvimento do psicoterapeuta e o modelo de treinamento, sendo que o primeiro deve abranger as modificações que surgem no sujeito durante seu treinamento, enquanto que no segundo caso, o modelo de treinamento deve prescrever ações concretas no contexto da supervisão que facilitem as mudanças no supervisionado.

O cuidado com as limitações dos modelos é um dever do supervisor, pois como indicam Ellis, Dell e Good (1988), os modelos de supervisão, assim como as diversas teorias psicoterápicas, respondem a todas as dimensões do processo de forma separada, o que limita a validade do próprio modelo baseado nestas.

A partir da literatura, a classificação mais freqüentemente utilizada considera que os modelos de supervisão mais freqüentes estão baseados em duas posições: nas teorias psicoterápicas ou nas propostas desenvolvimentistas (Bernard e Goodyear, 1992).

Os modelos baseados nas teorias psicoterápicas tem em comum a definição do treinamento de um profissional competente a partir dos pressupostos clínicos do modelo e associar o desempenho/papel do supervisor ao papel do clínico. No modelo psicanalítico, por exemplo, a competência do profissional é verificada a partir da capacidade de estabelecer relações transferenciais e contra-transferenciais com seu cliente, de tal forma que os processos de identificação sejam claros para o terapeuta (Bernard e Goodyear, 1992).
O objetivo da supervisão é o "instrumento analítico" do supervisionado, sendo que isto seria ocorreria a partir da aprendizagem do supervisionado em escutar o paciente, compreender a sessão e elaborar as intervenções adequadas. $O$ supervisor e o supervisionado devem conjuntamente proceder uma avaliação mútua da relação de trabalho, avaliação do progresso do paciente, reavaliação da terapêutica proposta e reconhecimento e manejo das situações que limitam a aprendizagem (Brustein e Boft, 1991).

Para Silva (1991, pg. 280), "o supervisor usará sempre um modelo de supervisão baseado em seu modelo de análise, em suas experiências de supervisão e na teoria que desenvolveu e teceu ao longo curso de sua vida...". Pela posição de Silva, o modelo de supervisão em psicanálise estáe sempreestará posicionado em uma relação de caráter clínico entre supervisor e supervisionado. Esta posição parece alienar tanto supervisor como supervisionado dos avanços conquistados por outras formulações teóricas, até mesmo da própria teoria caso o supervisor não esteja sempre em dia com os avanços do seu enfoque, o que ocorre muitas vezes no Brasil. Tal situação talvez encontre explicações na visão de Brustein e Boft (1991), que postulam que o modelo de supervisão psicanalítico é transmitido de pai para filho, de geração para geração, ou seja de supervisor para supervisionado.

Para Bernard e Goodyear (1992), no enfoque comportamental, ao contrário, a competência que se objetiva, é descrita em termos do domínio dos princípios da aprendizagem (conhecimento e uso), enquanto que o papel do supervisor se direciona mais para o de professor. O supervisor atua baseado nos conceitos de comportamentos adaptados e mal-adaptados, atuando em termos de aprendizagem e contingências, consequentemente usando princípios e procedimentos já testados. Os autores afirmam, ainda, que não será surpresa se o supervisor comportamental for mais sistemático que os supervisores de outros enfoques na 
preservação de suas metas e processo de supervisão. Certamente isto é esperado, posto que se espera um planejamento sistemático, definições operacionais, avaliações constantes e atividades similares.

Para Bootzin e Ruggill (1988), o treinamento em Terapia Comportamental deve abarcar o ensino didático em Psicologia Experimental, Teoria da Aprendizagem e Modelação ao vivo, por escrito ou vídeo-tape das habilidades clínicas, além de reforçamento e simulações, enquanto que Collins, Foster e Berler (1986), defendem a idéia de que as possibilidades do treinamento em Psicologia Comportamental são fundamentadas, principalmente, em três estratégias: treinamento baseado nas técnicas, na teoria ou no método científico. De um modo geral, dentro de uma perspectiva comportamental-cognitiva de supervisão, tanto terapia como supervisão são vistos como reeducação ou reaprendizagem (Schmidt, 1979).

No modelo rogeriano, um dos pontos primordiais do treinamento do futuro terapeuta está nas habilidades interpessoais inerentes ao exercício da psicoterapia. Tais habilidades são descritas como uma variedade de atitudes facilitadoras e de características interpessoais que devem ser desenvolvidas pelo psicoterapeuta que atua dentro desta proposta: empatia, congruência, respeito e simpatia (Lambert e Arnold, 1987). O supervisionado deve trabalhar muito para conseguir a habilidade e motivação para crescer e explorar a situação terapêutica e o seu self (Bernard e Goodyear, 1992).

Entretanto, Goodyear, Abadie e Efros (1984) afirmam que deve-se considerar que a orientação teórica dos supervisores pode pré-determinar seus comportamentos, papéis e atitudes, delineando, assim, o perfil do estilo de supervisão de uma abordagem teórica. Estes mesmos autores realizaram um estudo neste sentido, que contou com a colaboração de quatro grandes psicólogos e supervisores norte-americanos: A. Ellis (Terapia
Racional-Emotiva), C. Rogers (Abordagem Centrada na Pessoa), E. Polster (Gestalt-Terapia) e R. Ekstein (Psicanálise). Os resultados indicaram que Rogers e Polster focalizaram mais os aspectos individuais/ experienciais do supervisionado e/ou seu cliente, enquanto Ekstein focalizou mais a conceitualização do caso, e Ellis, o ensino de habilidades e técnicas específicas, dados que estavam de acordo com o esperado.

Holloway e cols. (1989), ao estudarem os efeitos da orientação teórica sobre a forma de poder e envolvimento na supervisão, utilizando os dados referentes a uma supervisão sobre um mesmo caso simulado registrada em videoteipe, com cinco grandes teóricos/ supervisores (N. Kagam, E. Polster, C. Rogers, A. Ellis e R. Ekstein) verificaram, através da análise do discurso entre supervisor e supervisionado, que os padrões de envolvimento e poder eram semelhantes aos previstos pela posição teórica de cada supervisor, indicando uma coesão interna e validando o estudo de Goodyear, Abadie e Efros (1984).

Outro estudo conduzido por Putney, Worthington e Me Cullough (1992), comparou os efeitos da orientação teórica de supervisores e supervisionados sobre a percepção da supervisão, foi identificado que os supervisores das teorias humanista e psicodinâmica enfatizam mais o relacionamento com seus supervisionados que os supervisores comportamentaiscognitivos. Ao mesmo tempo, estes supervisores assumem mais o papel de consultor que seus colegas humanistas e psicodinâmicos, que tendem a assumir o papel de psicoterapeuta durante a supervisão. No tocante ao desenvolvimento das habilidades clínicas e estratégias de intervenção, os supervisores comportamentais-cognitivos enfatizam mais este aspecto, enquanto que os humanistas e psicodinâmicos objetivam a conceitualização da dinâmica do cliente.

A questão central é a possibilidade real de se encontrar elementos comuns a todas as 
propostas teóricas que permitam uma maior eficácia das diversas estratégias de treinamento.

Desta forma, face à informações apresentadas anteriormente, o foco desta pesquisa direcionou-se para a verificação dos estilo e foco de supervisão adotados pelos supervisores de Psicologia clínica na realidade brasileira. Mais especificamente, são descritos a seguir os objetivos que nortearam a realização do presente estudo:

a) verificar quais são os estilos de supervisão adotados;

b) identificar as principais dimensões focalizadas pelo supervisor; e

c) comparar supervisores que atuam em abordagens teóricas distintas (Cognitivo-Comportamental e Psicodinâmica) afim de elaborar um perfil por linha teórica.

\section{MÉTODO}

\section{Sujeitos}

Foram sujeitos, 18 supervisores de estágios clínicos de instituições da grande São Paulo e região, independente de linha teórica, experiência ou sexo. Os sujeitos foram determinados acidentalmente, sendo facultado o direito à não participação dos mesmos neste estudo.

Dos sujeitos-supervisores, $61 \%$ eram do sexo feminino $(n=11)$ e $39 \%(n=7)$ do sexo masculino. Os mesmos possuíam idade média de 40,5 anos, com 13,5 anos em média de graduados, variando de 8 a 21 anos de tempo de graduação. A experiência média de supervisão era de 7,5 anos, variando entre 2 a 21 anos, sendo que, no tocante ao tipo de instituição que os sujeitos cursaram na graduação, verificouse que $83 \%$ cursaram instituições particulares ( $N=15)$ e 17\% estudaram em instituições públicas $(\mathrm{N}=3)$.

Em relação à formação em nível após a graduação (especialização ou pós-graduação), a totalidade dos sujeitos referiram já terem cursado ou estarem cursando algum tipo, sendo que isto ocorreu em diversos níveis, sendo predominante o "mestrado completo" (33,33\%), seguido pela "especialização completa" (27,77\%), "mestrado incompleto" (22,22\%), "doutorado incompleto" $(11,12 \%)$ e "doutorado completo" (5,56\%).

Quanto à linha teórica que os sujeitos atuam, os mesmos foram divididos em dois grandes grupos teóricos: grupo psicodinâmico e grupo cognitivo-comportamental. Dos sujeitos, $67 \%$ eram do grupo psicodinâmico ( $\mathrm{N}=12)$ e $33 \%$ do grupo cognitivo-comportamental $(\mathrm{N}=6)$. Todos os sujeitos supervisionavam tendo como base seu próprio referencial teórico.

\section{Material}

Dentre os vários instrumentos encontrados, os escolhidos referiram, segundo Bernard e Goodyear (1992), um apoio maior na literatura. Mais especificamente, foram utilizados os seguintes instrumentos:

1- Inventário de Estilo de Supervisão: escala do tipo Likert, abrangendo 34 itens relativos aos estilos de supervisão, tais como humor, explícito, etc. Apresenta-se em duas formas equivalentes, uma para o supervisor outra para o supervisionado, tendo sido usada apenas a versão para supervisor. Possui guia de correção e foi desenvolvido por Friedlander e Ward (1984, apud Bernard e Goodyear,1992), traduzido, revisto e adaptado por Campos (1994). Este instrumento está dividido em três categorias:

I- Atrativo: relativo a um estilo de supervisão baseado na atração interpessoal, e de caráter agradável.

II-Sensível: relativo a um estilo de supervisão cuja relação é sensível às necessidades do supervisionado (pessoais e/ou profissionais). 
III- Orientado para a Tarefa: indica um caráter mais prático da supervisão, que está voltada para as tarefas a serem realizadas nos atendimentos e na eventual formação teórica complementar.

2-Questionário de Foco e Estilo de Supervisão Para Supervisores: escala tipo Likert, dividida em três partes: Personalidade (com 18 itens), Foco de Supervisão (com 20 itens) e Estilo de supervisão (com 20 itens). Possui chave para sua correção e foi desenvolvido por Vager, Wilson, Brewer e Kinnetz (1989, apud Bernarde Goodyear, 1992), traduzido, revisto e adaptado por Campos (1994). Este instrumento foi especialmente elaborado para avaliar o que o supervisor focaliza durante a supervisão e o seu estilo de supervisionar. $O$ instrumento está dividido em três níveis:

Sub-escala de Personalidade:

Afeto: necessidade de relacionamento afetivo expressada ou desejada pelo supervisor.

Inclusão: necessidade de inclusão no grupo de supervisão expressadaou desejada. Controle: necessidade de controle do processo de supervisão e psicoterapia expressada ou desejada.

Sub-escala do Foco de Supervisão:

Processo: supervisor focaliza a supervisão no processo e temas qualificados.

Conceitualização: o supervisor focaliza a supervisão sobre a conceitualização teórica da problemática do cliente.

Personalização: supervisor focaliza a supervisão em dimensões pessoais do supervisionado.

Sub-escala de Estilos de Supervisão:

Ensino:estilo do supervisor é didático/instrucional, ensinando habilidades ou técnicas para os seus supervisionados.
Terapêutico: estilo do supervisor é terapêutico/experiencial, enfocando mais aspectos dos impactos do atendimento no componente pessoal do supervisionado.

Consultor: estilo do supervisor é de atuação como um par do supervisionado, atuando apenas quando solicitado.

\section{Procedimento}

O procedimento foi organizado de modo a evitar qualquer contato entre sujeitos e 0 pesquisador, pois desta forma o sigilo fica meIhor garantido.

No primeiro momento, o pesquisador obteve a autorização das instituições de ensino para a coleta de dados. Nesta ocasião, o número de supervisores de estágios clínicos da instituição era solicitado, assim como a linha teórica de trabalho de cada um dos possíveis sujeitos, o que foi posteriormente confirmado com cada sujeito.

A seguir, um funcionário administrativo da clínica-escola era instruído para entregar os instrumentos aos supervisores. Os instrumentos foram acompanhados de uma carta do pesquisador que continha as informações sobre a pesquisa e o telefone do pesquisador para um eventual contato. O supervisor era orientado para devolver o instrumento no prazo de até 45 dias em caso de sua participação para a mesma funcionária administrativa da clínica- escola. Em caso da recusa da participação, o instrumento deveria ser devolvido, evitando que o pesquisador ficasse no seu aguardo.

Após 15 dias do prazo estipulado para retorno voluntário do instrumento, o Autor entrou em contato telefônico com as instituições para averiguar se mais algum instrumento tinha retornado. Foi garantido sigilo das informações levantadas a todos os sujeitos e instituições. 


\section{RESULTADOS}

A análise estatística ${ }^{1}$ dos resultados recorreu aos Testes de Dependência_viabilizadas pelo Teste $T$ de Wilcaxan e o Teste Ude MannWhitney, trabalhando-se sempre ao nível de 0,05 , além do Teste de Correlação de Spearman, que foi analisado dentro da mesma margem de erro que os testes anteriores (Siegel, 1956).

Embora o grupo. Cognitivo-Comportamental tenha seis sujeitos e o grupo. Psicodinâmico onze, nos cálculos estatísticos o número de sujeitos varia, pois ocasionalmente os sujeitos não responderam os instrumentos de modo completo.

\section{INVENTÁRIODEESTILOSDESUPERVISÃO}

No grupo Cognitivo-Comportamental, a média verificada foi em "Atrativo" obteve a média de 4,97 pontos, em "Sensível" o resultado aferido foi 5,60 pontos e em "Orientada para a tarefa" 4,83 pontos.

Tabela 1 - Comparação intra-grupo Cognitivo-Comportamental $(\mathrm{N}=6)$ nas provas de Correlação de Spearman $(n=6 ; n$. sig. $=0,05)$ e o Teste T de Wilcoxon $(n=6 ; n$.sig. $=0,05)$ no Inventário de Estilo de Supervisão.

\begin{tabular}{l|l|l|l|l|l}
\hline \multicolumn{1}{c|}{ Comparação } & $\mathrm{r}_{0}$ & $\mathrm{r}_{\mathrm{c}}$ & $\mathrm{T}_{0}$ & $\mathrm{~T}_{\mathrm{c}}$ & Decisão \\
\hline Atrativo vs. Sensível & 0,87 & 0,71 & 0 & 0 & Ho rejeitada \\
\hline Atrativo vs. Orientado $\mathrm{p} /$ tarefa & 0,41 & 0,71 & 7 & 0 & Ho não rejeitada \\
\hline Sensível vs. Orientado $\mathrm{p} /$ tarefa & 0,67 & 0,71 & 1 & 0 & Ho não rejeitada \\
\hline
\end{tabular}

Na tabela 1, encontra-se as resultados da comparação intra-grupa das sujeitos cognitiva-comportamentais. No teste de correlação de Spearman, observou-se uma correlação significativa entre o desempenho dos sujeitos nas categorias "Atrativa" e "Sensível" $\left(r_{0}=0,87\right.$ para $\left.r_{c}=0,71\right)$ enquanto que o mesmo não se observou nas comparações entre "Atrativa" e "Orientada para a tarefa" $\left(r_{0}=\right.$ $0,41)$ e entre "Sensível" e "Orientada para a tarefa" $\left(r_{0}=0,67\right)$. A relação entre as categorias "Atrativa" e "Sensível" demonstraram que nos itens destas sub escalas, as desempenhos apresentaram a mesma tendência.

Na comparação do desempenho através do teste de Wilcoxon (Tabela 1), observou em
"Atrativo vs. Sensível" o valor igual a zero, o que levou a hipótese nula ser rejeitada $\left(T_{c}=0\right)$, o que significa que o desempenho dos sujeitos nestas duas categorias tendeu a ser significantemente diferente. Em "Atrativo vs. Orientado para a tarefa" a hipótese nula não foi rejeitada, com T observado igual a sete. A mesma situação ocorre em "Sensível vs. Orientado para a tarefa" onde o T observado foi igual a um, levando à não rejeição da hipótese nula.

No grupo Psicodinâmico o estilo "Atrativo" obteve média de 4,49 pontos, enquanto que "Sensível" registrou 5,37 pontos e "Orientado para a tarefa" 4,81 pontos.

\footnotetext{
(1) Vale destacar que os testes de dependência analisam a distribuição das respostas dos sujeitos e a prova de correlação analisa a relação das respostas através dos postos.
} 